\title{
A classical presentation of Peyronie's disease
}

\section{Ralph Drosten}

MB BCh, FCRad Diag

Department of Thoracic Imaging Brigham and Women's Hospital Boston, USA

\section{Case presentation}

A 47-year-old man presented to his urologist complaining of painful erections that had increasingly deviated to the right over the previous 6 months. He experienced some difficulty, but no pain, during intercourse. No episode of trauma was recalled. A 'hardness' related to the subcutaneous tissue of the dorsum of the penis was noted 2 months prior to this presentation. Extension towards the glans occurred over the intervening 2 months.

Clinical examination confirmed a mobile, non-tender, firm plate of tissue, tracking subcutaneously along

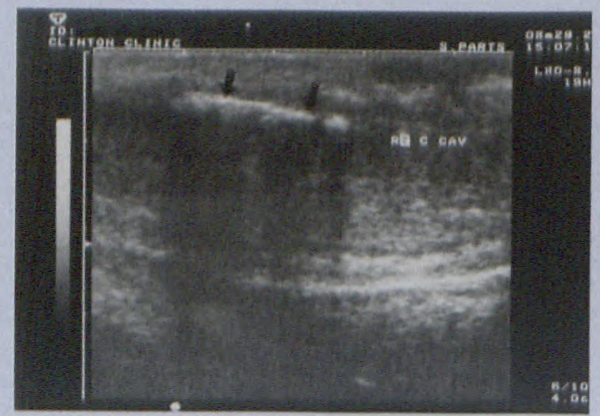

Figs 1 and 2. Saggital and axial ultrasound images showing tubular calcification (arrows) and associated acoustic shadow.

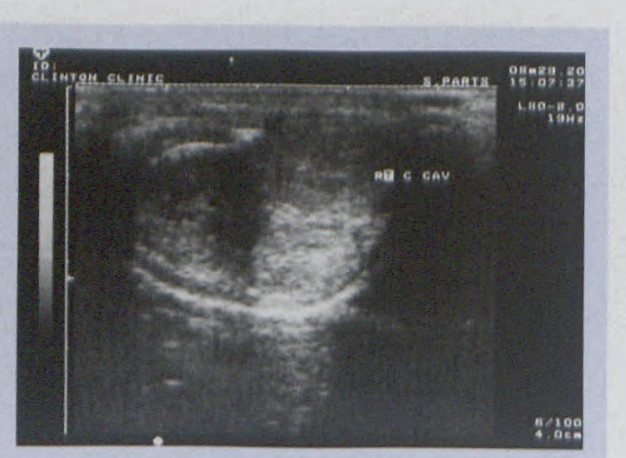

the dorsum of the penis, from the base to within $10 \mathrm{~mm}$ of the glans.

High-resolution ultrasound examination (Fig. 1. - saggital plane, and Fig. 2. - axial plane) confirmed a sheet of calcification throwing an acoustic shadow, tracking over the dorsal surface of the mid-right corpus cavernosum. No soft-tissue mass or architectural distorsion was associated. The corpora cavernosa and the corpus spongiosum were normal and symmetrical, bilaterally.

A soft-tissue exposure lateral X-ray of the penis (Fig. 3) confirmed sharply defined tubular calcification in the subcutaneous tissues of the dorsum of the penis.

A French surgeon, François de la Peyronie, first described this ailment in 1743 . The problem was noted in print as early as 1687 . Early writers classified it as a form of impotence. Experts now recognise impotence as (1)

(1)

(1)

\section{Discussion}

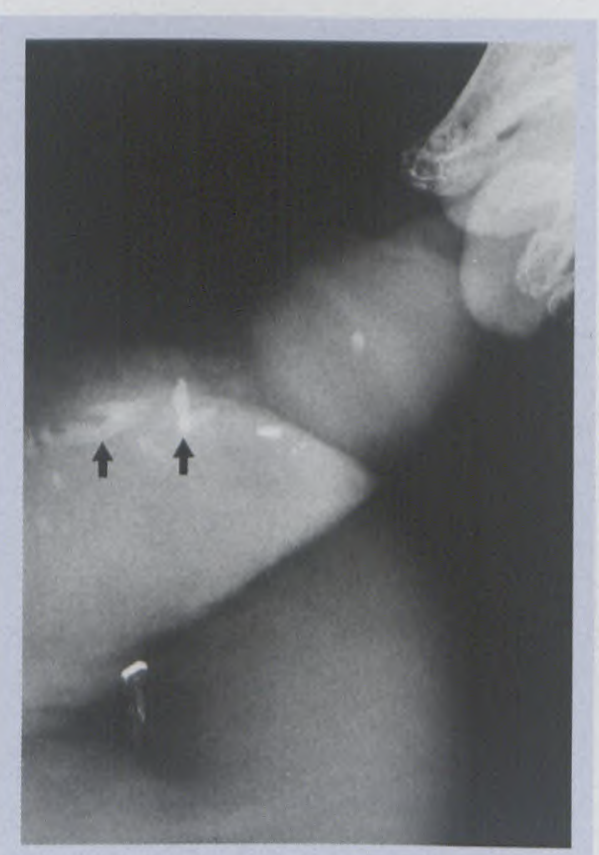

Fig. 3. Lateral soft-tissue $X$-ray of the penis confirming tubular calcification related to the dorsum of the penis (arrows).

one factor associated with the disease, a factor that is not always present. ${ }^{1}$

Men with Peyronie's disease usually seek medical attention because of difficulty experienced during intercourse - the presenting feature of our patient - and painful erections. ${ }^{2}$

Peyronie's disease is classically described as induration of the cavernous bodies of the penis, causing fibrosis. It often occurs in a mild form that heals within 6 - 15 months, without treatment. In severe cases, the hardened plaque reduces flexibility, causing pain, and forcing the penis to bend or arc during erection. About $30 \%$ of men with Peryonie's disease develop fibrosis in other elastic tissues, such as the hand (Dupuytren's contracture) and foot. Familial factors have also been postulated. ${ }^{3,4}$

Some believe that the plaque of Peyronie's disease develops following local trauma. ${ }^{1-3}$ The traumatised area may heal slowly or abnormally in the case of recent trauma, or due to the 
minimal amount of blood-flow in the sheath-like fibres. In cases that heal, the plaque does not advance beyond the inflammatory phase. In cases that persist, the plaque undergoes fibrosis, or even calcification.

While trauma might explain acute cases of Peyronie's disease, it does not explain why most cases develop slowly and with no apparent traumatic event..$^{5}$ It also does not explain why some cases disappear quickly, and why similar conditions such as Dupuytren's contracture do not seem to result from significant trauma.

\section{Imaging}

High-resolution ultrasound is the initial imaging modality of choice. ${ }^{1}$ It clearly demonstrates the corporal bodies, corpus spongiosum and tuni$\mathrm{ca}$ albuginea. The cavernous bodies should have a homogeneous, uniform echotexture. The findings of hyperechoic foci or calcification within the corpora cavernosa or spongiosum may represent a Peyronie's plaque (Figs 1 and 2).

Computerised tomography (CT) is rarely indicated, but its excellent contrast and anatomical resolution better demonstrates the extent of the disease. ${ }^{1}$ It is more sensitive in the assessment of calcification than plain films. The corpora cavernosa are usually seen as round structures of homogeneous intensity, surrounded by a thin hyperdense margin - the tunica albuginea. In patients with Peyronie's disease, areas of diminished density and thickness of the tunica albuginea secondary to inflammatory oedema are noted. Calcified areas are also well seen (CT is more sensitive than ultrasound with regard to calcification). CT can show disease that cannot be palpated or demonstrated clinically. A diffuse increase in the thickness of the tunica albuginea indicates generalised disease of the entire penis.

Magnetic resonance imaging (MRI) clearly demonstrates changes in the tunica albuginea and corpora cavernosa, as well as the anatomical relationship between the plaques and the penile arteries.

\section{Treatment}

Since the cause and progression of the disease are not well understood, the disease is treated empirically, i.e. methods that appear to help are persisted with. The goal of therapy is to keep the Peyronie's patient sexually active. Providing education about the disease and its course is often all that is required.

The plaque of Peyronie's disease often shrinks or disappears without treatment, and medical experts suggest waiting 1 - 2 years, or longer, before attempting to correct it surgically. During that wait, patients are often willing to undergo treatments that have unproven effectiveness. Our patient is currently being treated conservatively.

\section{Conclusion}

Peyronie's disease is a condition with prominent psychosocial implications. It offers a clinical management challenge. Ultrasound, and occasionally CT assessment of the penis delineates the extent of the disease and contributes to the monitoring of disease progression and resolution.

\section{References}

1. Resnick D, Boutin RD, Andersson I. Encyclopaedia of Medical Imaging. ISIS Medical Media, 1999: 474-475.

2. http://www.niddk.nih.gov/health/urolog/ pubs/peyronie/peyronie.htm

3. http://www.urologychannel.com/peyronies/ index.shtml

4. http://www.aafp.org/afp/990800ap/549.html

5. http://www.umm.edu/urology info/peyroni.htm 